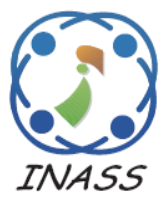

\title{
An ECG Denoising Method Based on Hybrid MLTP-EEMD Model
}

\author{
Mala Sinnoor $^{1 *} \quad$ Shanthi Kaliyil Janardhan ${ }^{2}$ \\ ${ }^{1}$ Department of Electronics and Communication, \\ Dr. Ambedkar Institute of Technology, Bengaluru, Karnataka, India \\ ${ }^{2}$ Department of Medical Electronics, \\ Dr. Ambedkar Institute of Technology, Bengaluru, Karnataka, India \\ * Corresponding author's Email: malasinnoor.ec@ drait.edu.in
}

\begin{abstract}
The Electrocardiogram signal (ECG) is highly susceptible to the electrical environment from where the motion artifacts were being recorded. The accurate representation of the ECG signal will necessitate removing the noise from various sources thereby resulting in a noise-free model. This present research work uses Multi scale Local Polynomial Transform (MLPT) technique that provides wavelet transform as an alternative when the MLPT model was non-equispaced. The proposed research combines two approaches such as MLPT and Ensemble Empirical Mode Decomposition (EEMD) forming a Hybrid Transform Model which is used for denoising in the research. The present research work considers white Gaussian noise and experimental results are based on the MIT-BIH Physionet Database. The results of the proposed hybrid MLPT-EEMD obtained better SNR values of $25.93 \mathrm{~dB}$ when compared to the existing Empirical Mode Decomposition technique (EMD) of 5.43dB, EMD with adaptive switching mean filter obtained 9.135 dB and S-Transform based Time-Frequency Filtering Approach obtained $13.82 \mathrm{~dB}$.
\end{abstract}

Keywords: Electrocardiogram, Ensemble empirical mode decomposition, Multi scale local polynomial transform, Denoising, Intrinsic mode function.

\section{Introduction}

Heart disease can be diagnosed widely using ECG signals and in clinical practice, the ECG signals are considered the most important tool to diagnose heart disease [1]. Therefore, it is very important to analyze and process ECG signals to detect heart disease. The different types of noises are introduced during the acquisition of ECG signals such as Electromyogram (EMG) noise caused by the movement and contraction of human muscles, electrode motion noise due to patient's sudden body movement and movement of the patient [2, 3]. Similarly, the white Gaussian noise in the channel is introduced during transmission of ECG signals through a channel, power line interference is because of improper grounding of the ECG machine. The ECG waveform will be distorted due to the introduction of these different noises and also disturb the features for diagnosis. Therefore, the diagnosis of cardiac disorders at the early stage is necessary for giving proper medication to arrhythmia patients [4]. The ECG signals are a well-established tool used in medical assessment for monitoring the electrical activity of the heart. Electrocardiogram (ECG) is a non-stationary, non-linear and in-homogeneous tool that can analyze different types of arrhythmia and perform visual interpretation from ECG recording. The process of performing the visual interpretation will be difficult for the cardiologists and hence various researches were undergone for performing denoising of ECG signals.

The denoising approach such as morphological filtering approach, wavelet based approach, empirical mode decomposition (EMD), Adaptive filtering method has been utilized for various researches [5]. The morphological filtering technique is used effectively that limited the interference signal but faced issues such as high-frequency interference signal and a truncation error [6]. Also, the filter quality can be adjusted automatically according to the 
noise characteristics by the adaptive filtering (AF) method. Various ECG signals are addressed with the aid of an adaptive signal extraction method which makes use of discrete wavelet transformation mixed with the adaptive parameters [7].

The classification results obtained from the proposed modified CNN into 4 types of beats such as atrial fibrillation (AF), ventricular fibrillation (VF), ventricular tachycardia (VT), supra ventricular tachycardia are resulted and are obtained for determining the arrhythmia type present. Thus, analyzing the proposed method effectiveness of the proposed method improved the SNR, MSE rate better when compared with the denoising algorithm as per the previous denoising techniques used. The proposed technique will solve the shortcoming of the first order IMF disorder better when compared with the traditional EMD denoising technique improves in terms of hard threshold function and traditional soft function $[9,10]$

Thus, to overcome the problems that occurred in the existing approaches to perform denoising, a hybrid transform model combined Multiscale local polynomial technique (MLPT) and EEMD is model. The developed model discarded the first-order IMF in the conventional EMD denoising approach and a new threshold denoising function was developed. This model enhanced the conventional soft and hard threshold functions. The EEMD method has the ability to scale better as evidenced by trials with the addition of white noise to the signal, the resulting IMF does not show links with other IMF. The computation of IMF components obtained will evaluate the average value among the blocks. The ternary pattern will be adopted that reflects the differences among the threshold and the pixels.

The performance of the proposed algorithm is examined on the MIT-BIH arrhythmia database with white Gaussian noise and evaluated based on mean square error (MSE), peak signal to noise ratio (PSNR) and signal to noise ratio (SNR). The results are compared with EMD, sample entropy and improved threshold function technique and the proposed method show better SNR.

The structure of the paper is as follows: section 2 describes about the existing methods involved for denoising approaches and also Arrhythmia disease detection. Section 3 discusses about the proposed method, section 4 discusses about the results and discussion. The section 5 discusses about the conclusion and future work.

\section{Literature review}

Manas Rakshit [2] developed an empirical mode decomposition and adaptive switching mean filter (ASMF) for performing ECG denoising effectively. The ASMF and EMD both exploited reduced noises in the ECG signals based on the distortion. However, the developed approach was harmed with the highfrequency details ignored the noises for QRS complex regions. The conventional EMD based methods failed to reject high frequency noises and also to preserve the QRS complexes.

Wang [11] developed a deep learning approach for atrial fibrillation signals classification based on convolutional and modified Elman neural network (MENN). The developed network structure was primarily stacked by $\mathrm{CNN}$ and MENN, while automatically performed end-to-end signals classification. Moreover, tenfold cross-validation was employed to evaluate the classification performance of the model on the MIT-BIH AF database. The model involved the higher computational cost, and was not applicable for practical application.

Murugappan [12] predicted the sudden cardiac arrest (SCA) based on the ECG Morphological Features and the support vector machine. The developed model confirmed that the sample entropy features were effectively predicted sudden cardiac arrest (SCA) and Ventricular fibrillation onset. The developed model used the SVM classifier obtained maximum classification rate. However, the developed model used dataset of smaller size for the prediction of SCA algorithm. However, the extensive database for analyzing the generalization ability of the developed algorithm for SCA prediction was difficult which lowered the values of SNR.

Dengyong Zhang [13] developed an efficient ECG denoising approach using empirical mode decomposition model that showed improvement in improved threshold function and sample entropy. The developed model solved the problem of shortcoming by discarding the IMF first order using traditional EMD denoising approach improved the threshold functions. The developed model utilized MIT-BIH database where three sort of noises were simulated such as gaussian noise, electromyogram (EMG), and power line interference. The developed model solved the shortcoming and discarded the first order IMF with traditional EMD denoising approach. The developed model concentrated mainly on white Gaussian noise but neglected to simulate power line interference and EMG signals which impacted on the SNR. 


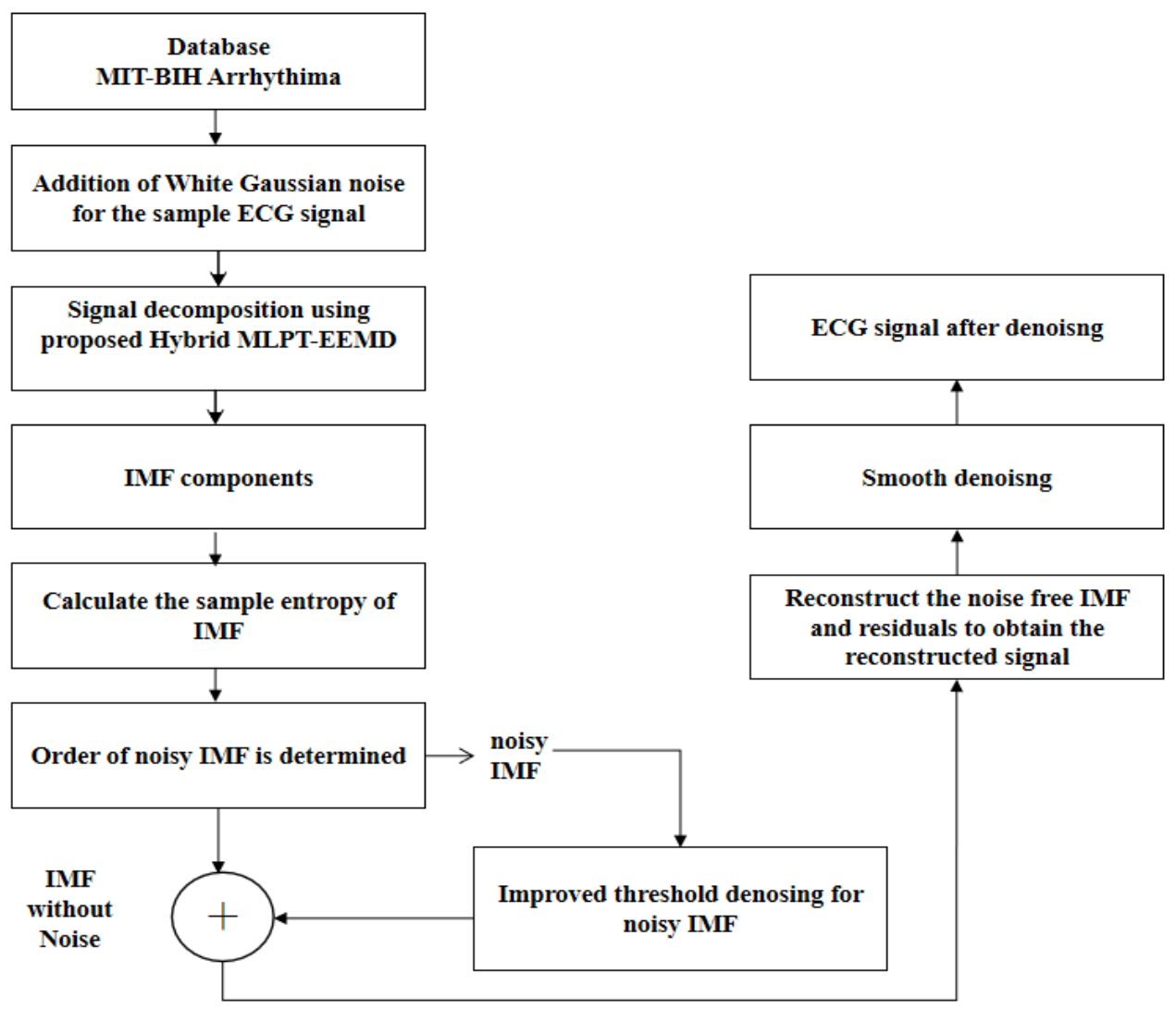

Figure. 1 Block diagram of the proposed method

Ankita Mishra [14] developed S-Transform based time frequency based filtering approach for ECG signal. The morphological process was employed visualized the ECG spectrum signals based on Stransform based time frequency method. The time frequency plane was fitted on the surface eliminated the noise and layer threshold was used for eliminating. The developed model assessed various normal and abnormal ECG signals which were reliable reduced the noise with low levels of inputs that was dependent on the availability of the referenced signal.

\section{Proposed method}

The massachusetts institute of technology-beth israel hospital (MIT-BIH) database is considered for the research work, which consists of ECG signals. The ECG signals undergo pre-processing and the preprocessed signals are then processed for QRS peak detection from where the features are extracted. Fig. 1 shows the block diagram of the proposed method and the same is being dealt with in the details in upcoming sections.

\subsection{Data collection using MIT BIH database}

MIT-BIH arrhythmia database is used in the research which analyses the ECG signal that is available publicly for heart arrhythmia detection.
The ECG of 4000 Holter recordings are included in the MIT-BIH Arrhythmia Database which is obtained from 1975 to 1978 from beth israel hospital arrhythmia laboratory [15]. The sample of ECG signals with noises is shown in Fig. 2. The Fig. 2 (a) is the Patient record number of 100 and (b) is the Patient record number 103.

\subsection{Pre-processing as a denoising technique}

The ECG records contain different sorts of noises, artifacts that are recorded and captured by skin electrodes. The ECG signal usually consists of highfrequency noise such as electromyography (EMG) signal, power line interference, etc., and lowfrequency noise such as baseline wander. The preprocessing stage is performed to detect noise accurately so that different components can be extracted, which would play an important role in the further process. The Fig. 3 shows the pre-processed ECG signals where (a) represents Patient record number 100 and (b) Patient record number 103.

Multi scale local polynomial transform (MLPT) technique that provides wavelet transform as an alternative when the MLPT model was nonequispaced. The proposed research combines two approaches such as MLPT and ensemble empirical mode decomposition (EEMD) as a hybrid transform model to produce noise free ECG signals. The 


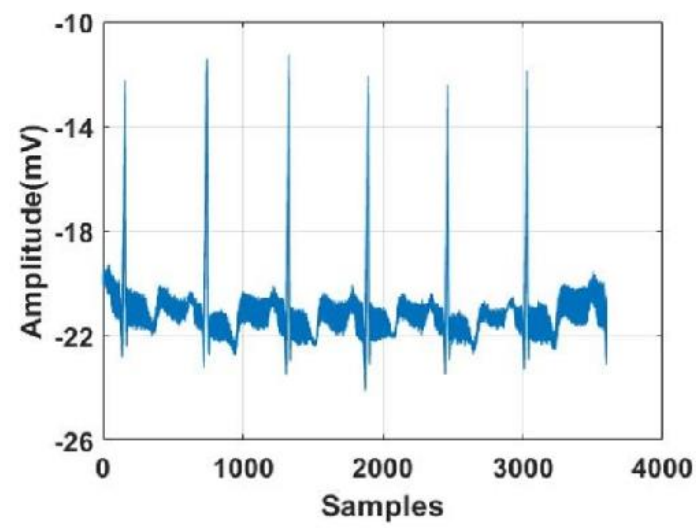

(a)

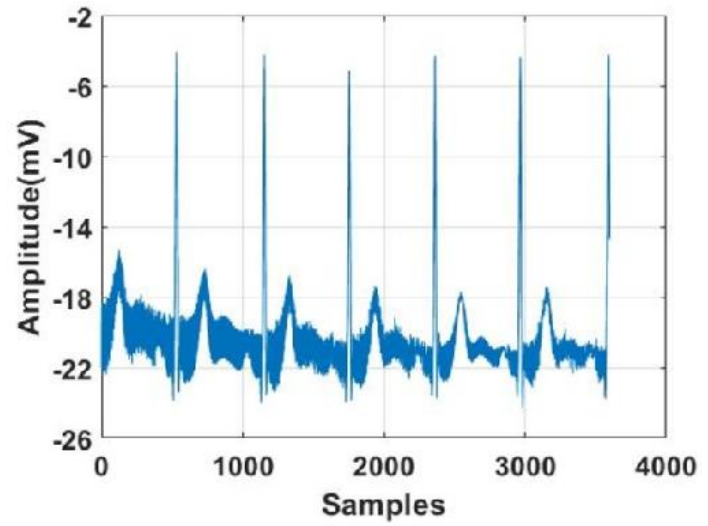

(b)

Figure. 2 The sample ECG signals with noise: (a) Patient record number 100, (b) Patient record number 103

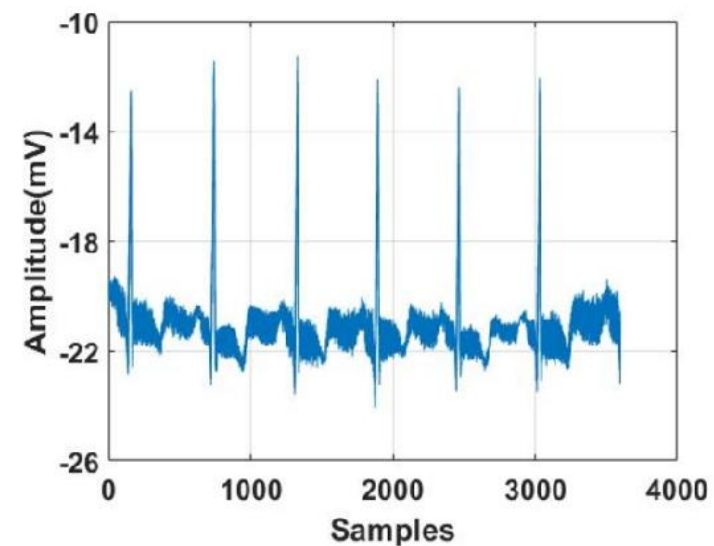

(a)

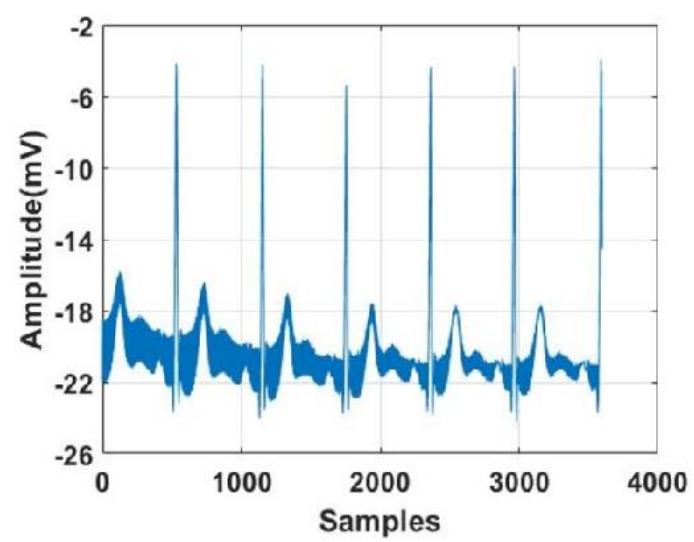

(b)

Figure. 3 Pre-processed ECG signals: (a) Patient record number 100 and (b) Patient record number 103

decomposition approach is conducted using the local polynomial approach gives rise to coefficients with respect to the local time. The scales present in the multi-scale transformation are set to be at the best by choosing the bandwidth with the sequence. The local polynomial approach will lead to representing the information of coefficients with respect to the local time. The multi-scale transformation will be set for choosing the bandwidth sequence. The bandwidth has a scale parameter that calculates the transform and there is a natural way to sample the data.

\subsection{Multi-scale local polynomial decomposition}

The MLPT is constructed based on the laplacian pyramid scheme that starts by vector assignment during the vector observations represented as $Y$ up to the finest scale vector $S_{j}$.

Where $j=J-1, J-2, \ldots, L$

From the above expression, $L$ is the last iteration of the laplacian pyramid scheme. The white noise is canceled from the collected signals where each signal is operating in a time-space ensemble mean. Thus, the signal will survive up to the final signal which will be added with the ensemble mean. There will be no finite or infinitesimal amplitude with the vital noise which forces an ensemble model in finding out all the possible solutions. The finite magnitude noise makes distinct scale signals residing as an IMF results from a significant mean which is calculated using Eq. (1).

$$
S_{j}=\left(\widetilde{H}_{j} s_{j+1}\right)_{e}
$$

Where $\widetilde{H}_{j}$ is known as the lower variance in $S_{j}$ and the index $e$ will stand for the subset of $\left\{0, \ldots, n_{j+1}-1\right\}, n_{j+1}$ is known as the vector length of $s_{j+1}$. The subset will not need the subset $e$ that consists of even numbers ranging from $\left\{1, \ldots, n_{j+1}\right\}$ that means $S_{j}=\left(\widetilde{H}_{j} S_{j+1}\right)$ which consists of the invertible matrix that has aimed for performing the data pre-processing at that instance [11]. The matrix $\widetilde{H}_{j}$ is a square matrix but not always required to be an invertible matrix and can be used as an anti-aliasing instance matrix. The present research work consider $\widetilde{H}_{j}=I_{n_{j+1}}$ which results with $s_{\mathrm{j}}=s_{j+1, e}$. If $e$ is required to set the even numbers then the coefficient level will give rise to $s_{\mathrm{j}, \mathrm{k}}=s_{j+1,2 k}$ which is calculated using the Eq. (2). 


$$
d_{j}=D_{j}^{-1}\left(s_{j+1}-P_{j} s_{j}\right)
$$

$D_{j}$ is an optional diagonal matrix for performing the process of standardization and normalization of the generated coefficients. $P_{j}$ is known as the smoothing matrix obtained from the local polynomial and the rows are filled with the $P_{j}\left(\mathrm{t}_{\mathrm{j}+1,2 \mathrm{k}+1} ; t_{j}\right) \cdot t_{j}$ refers to the grid locations of the covariance values.

The multi-scale local polynomial transform has the prediction matrix $P_{j}$ that cannot be used as a local polynomial and smoothing operation in the lifting scheme inverse transform the coefficients lead to reconstruction. The reconstruction can be understood from coarse-scale approximation from where all values result with zero. Therefore, the odd fine-scale coefficients follow from $S_{j+1, e^{\prime}}=P_{j} S_{j}+d_{j}=$ $P_{j} S_{j+1, e}$. To get smooth reconstruction, if an odd factor $t_{j+1,2 k+1}$ is near to the even neighbor then that should be the co-efficient. This is due to the inverse transform from processed coefficients that could cause a fractal-like reconstruction. Thus, the process of reconstruction will consider a rough scale approximation from where all details will be initially considered as zero. In that case, the odd fine-scale coefficients follow from $S_{j+1, e^{\prime}}=P_{j} S_{j}+d_{j}=$ $P_{j} S_{j+1, e}$. For easy reconstruction, it is necessary that if an odd factor $t_{j+1,2 k+1}$ is near to the even neighbor, then so should be the co-efficient which will be calculated using Eq. (3).

$$
\lim _{u \rightarrow t_{j, k}} P_{j}\left(u ; t_{j}\right) \cdot s_{j}=s_{j, k}
$$

Eq. (3) shows that $P_{j}\left(u ; t_{j}\right)$ is not a smoothing operation whereas wavelet transforms use lifting schemes which are more complicated [12]. If the wavelet transform is used for performing easy prediction, then interpolating part has to be considered apart from completeness. Another part is to perform easy predictions for interpolating from completeness. Thus, another difference among multiscale local polynomial and wavelets transforms scales has to be considered at every decision level. The prediction operator in a wavelet transform follows the gap between the adjacent points in $t_{j}$ and makes use of a fixed number of factors from $t_{j}$ that is near to the point $t_{j+1, e^{\prime}}$. The local sample density is used in the scaling of the prediction operation. The lifting scheme in 2D desires a triangulation or a few other systems which relate to the neighbourhood. In the local polynomial transform, the fixed bandwidth of scale and neighbourhood leads to a more stable decomposition which makes implementation easy. The non-zero numbers in row $k$ of the prediction matrix $P_{j}$ relay on the adjacent factors within the bandwidth around $t_{j+1,2 k+1}$. A matrix $P_{j}$ has a fixed number of non-zeros in a wavelet transform [13].

\subsection{Ensemble empirical mode decomposition}

The true and physical solution to the EMD is not only the one without noise but should be designated with the ensemble mean. As more trials are performed, the more will be the addition of noises to the signal. The present research work uses EEMD for the proposed research as it is important for improving the noise characteristics statistically [14]. The scale separation of the EMD is utilized by EEMD and allows the EMD method as a truly dyadic filter bank for all data. The mode mixing problem can be eliminated in EEMD by adding finite noise and preserving the physical uniqueness of decomposition. Therefore, the improved technique of EMD is represented as EEMD. The proposed method implied a signal which is the same as that of the noisy dataset contained the components IMF. The EMD method is very adaptive and the decomposition is achieved from the data. Eq. (4) shows the proposed EMD approach decomposes the data into $x(t)$ decomposed into IMFs that are represented $c_{j}$.

$$
x(t)=\sum_{j=1}^{n} c_{j} r_{n}+r_{n}
$$

In which $r_{n}$ is the residue of data $x(t)$, after $n$ quantity of IMFs is extracted. IMFs are easy oscillatory functions where frequency and amplitudes are varying.

The data amalgamation includes the signal and noise and thus the measurement of accuracy is needed for the powerful approach to collect and separate the observed data as each of them contains the noise. The main idea is to generalize this ensemble thought and to introduce it into the dataset represented as $x(t)$ and the observation made will be improved with the experiment once repeated many times. The white noise is random and has possible random noise encountered for measurement. Eq. (5) shows the conditions of those artificial observations for $i^{\text {th }}$ turn will be as follows:

$$
x_{i}(t)=x(t)+w_{i}(t)
$$

White noise $w_{i}(t)$ is added to every $i^{t h}$ observation results with a smaller signal-to-noise ratio but a uniform distributed scale is provided to EMD. Decomposition is not affected and mode mixing is avoided by enhancing EMD (EEMD) 


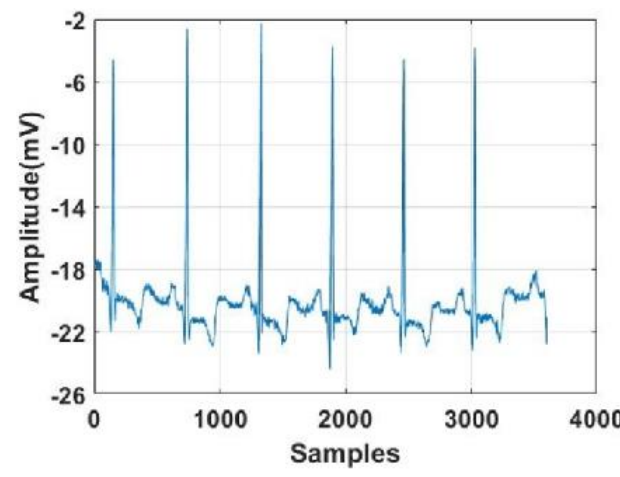

(a)

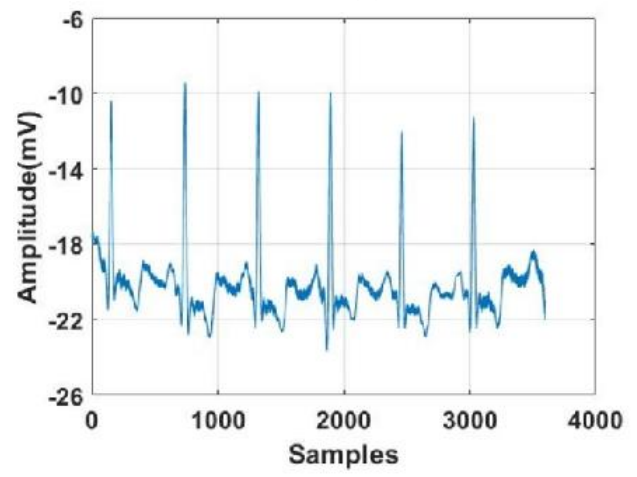

(c)

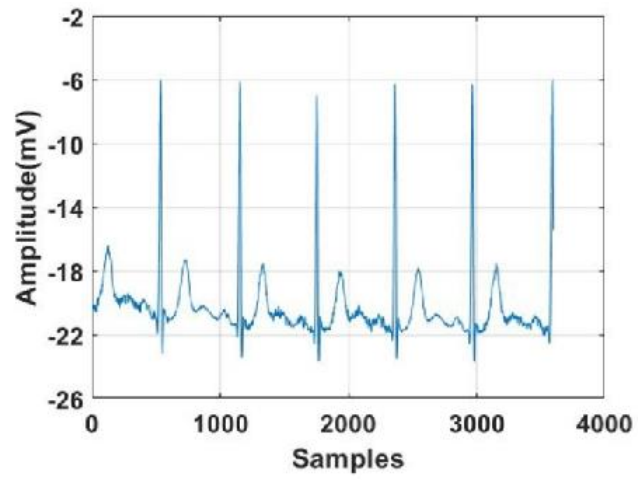

(b)

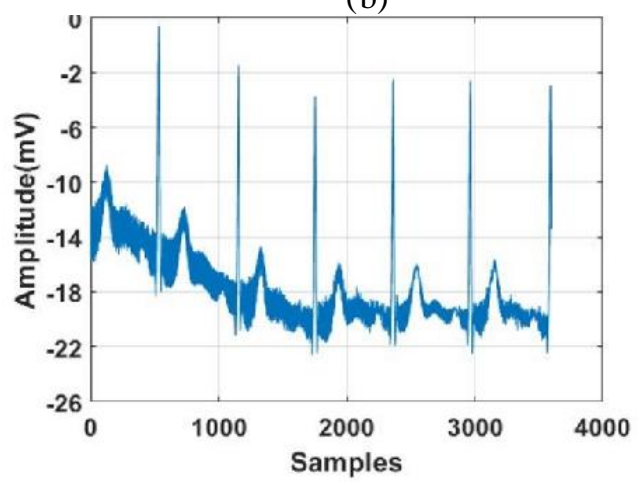

(d)

Figure. 4 The ECG signals obtained by using: (a) MLPT denoising approach for patient record number 100, (b) EEMD denoising approach for patient record number 100, (c)MLPT denoising approach for patient record number 103, and (d) EEMD denoising approach for patient record number 103

technique [15].

The procedure of the proposed EEMD is as follows:

1. The targeted data is added with a series of white noise

2. Added white noise to decompose with the data into IMFs;

3. The first and second steps are repeated simultaneously, but with distinct white noise series at each time; and

4. The final result can be obtained by the (ensemble) means of IMFs corresponding to the decomposition

The Fig. 4 (a) MLPT denoising approach for patient record number 100 Fig. 4 (b) EEMD denoising approach for patient record number 100 Fig. 4 (c) MLPT denoising approach for patient record number 103 Fig. 4 (d) EEMD denoising approach for patient record number 103.

EEMD utilizes various decomposition techniques as it adds white noise series that will decompose the data and will cancel the final mean of IMFs. The dyadic filter windows formed by mean IMFs reduces the chances of mode mixing. Fig. 3 shows the ECG signals obtained by using the MLPT denoising approach and EEMD denoising approach.
The EEMD will be implemented by the addition of white noise for the original data that has an amplitude of 0.1 of Standard Deviation is as shown in Fig. 4. The low-frequency components are determined but the high-frequency components will be hidden in the noise. The ensemble members were increased with numbers when the intermittent signals having higher frequency dominated. This intermittent signal resides under two EEMD components. This is due to neighboring IMFs' average spectra component overlapping the white noise components. Therefore, the mixture must obtain 2 adjutant components that form IMF and will check orthogonality conditions. If the condition for 2 IMF components grossly results in unorthogonal signal, then a single IMF with 2 components will be combined. The Fig. 5 shows the ECG signals obtained by using hybrid MLTP-EEMD denoising approach Fig. 5 (a) for patient record number 100 Fig. 5 (b) for patient record number 103.

This will result from EEMD to improve the extraction of data from the signals showed major enhancement in developing EMD technique for denoising.

\section{Results and Discussions}

The proposed hybrid denoising model was used for removing the noise and thus improved the 


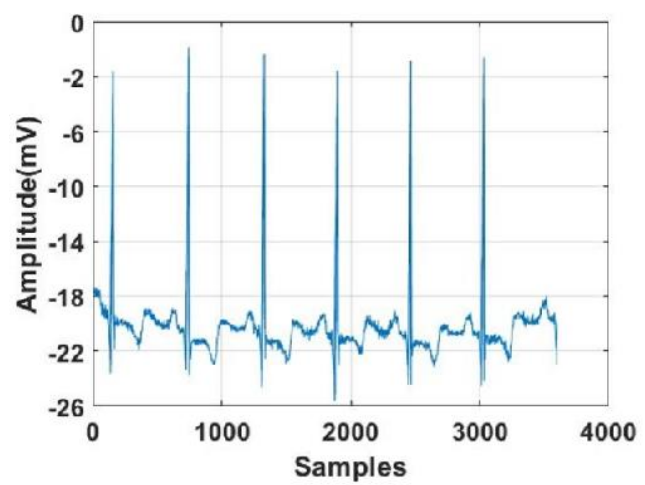

(a)

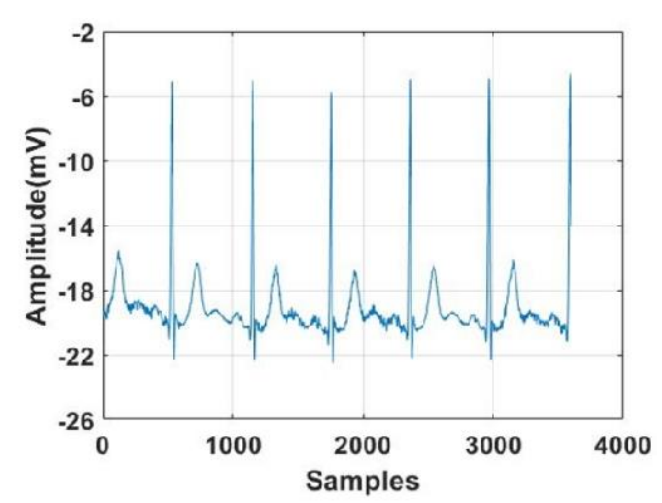

(b)

Figure. 5 The ECG signals obtained by using hybrid MLTP-EEMD denoising approach: (a) for patient record number 100 and (b) for patient record number 103

efficiency of the proposed model. The present research work utilizes MATLAB 2018a operating in i5 core processor. The present result section is divided into quantitative analysis and comparative analysis. The performance measures are used for evaluating the classification results using the following.

\subsection{Performance measures}

The results are evaluated in terms of mean squared error (MSE), signal to noise ratio (SNR), peak signal to noise ratio (PSNR). The mathematical expressions for all the performance measures are shown in Eqs. (6-8).

$$
\begin{gathered}
M S E=\frac{1}{n} \sum_{i=1}^{n}(y-\hat{y}) \\
S N R=\frac{P_{\text {signal }}}{P_{\text {noise }}} \\
P S N R=20 \cdot \log _{10}\left(M A X_{I}\right)-10 \cdot \log _{10}(M S E)
\end{gathered}
$$

\subsection{Quantitative analysis}

The present research work obtains the results that are evaluated in terms of MSE, PSNR, SNR. The results are shown for different ECG record samples obtained from MIT BTH for 7 different patients whose ECG records are 100, 103, 104, 105, 106, 115, and 215. The denoising methods such as MLPT, EMD are compared with the proposed hybrid MLPTEEMD model. The existing models showed higher MSE values compared to the proposed hybrid model. Similarly, the SNR and PSNR values were improved in the proposed hybrid model when compared to the existing models. Table 1 shows the MSE, SNR, and PSNR values with respect to the patient ECG record numbers 100,103,104,105,106, 115 and 215.

\subsection{Comparative analysis}

The existing models showed more MSE values compared to the proposed hybrid model. Similarly, the SNR and PSNR values were improved in the proposed hybrid model when compared to the existing models. Table 2 shows the MSE, SNR, and PSNR values to the patient ECG record number average values. The results showed that the proposed hybrid MLPT-EEMD obtained MSE values of 0.009161 and SNR values of 25.9307 and MSE of 0.0012 better. Similarly, with respect to $10 \mathrm{~dB}$ noise, the PSNR was obtained as $31.06 \mathrm{~dB}$ and MSE of 0.0012 better when compared to the existing empirical mode decomposition technique that achieved lesser MSE and SNR values of 0.0024 and $5.43 \mathrm{~dB}$. The CNN with modified elman neural network obtained MSE value of 0.0169 but the model was not reliable with respect to computation cost assessed various normal and abnormal ECG signals selected was reduced reliability. Whereas, the S-Transform based Time-Frequency filtering approach obtained SNR of 13.82 and MSE of 0.0287 that failed to reject high frequency noises and also to preserve the QRS complex.

\section{Conclusion}

This paper proposes an effective ECG denoising method based on a hybrid MLPT-EEMD transform model. The proposed work combines multi-scale local polynomial transform and ensemble Empirical mode decomposition to determine the order of IMFs and denoise the signal. In the traditional EMD denoising method, initially, the IMF components were discarded to remove high-frequency noises. Due to this, significant information was discarded and failed to achieve complete denoising. The robustness of the proposed denoising method is 
Table 1. The MSE, SNR, and PSNR values with respect to the patient ECG record number 100,103,104,105,106, 115 and 215 added with white gaussian noise $(20 \mathrm{~dB})$.

\begin{tabular}{|c|c|c|c|c|}
\hline $\begin{array}{c}\text { Patient } \\
\text { Record No. }\end{array}$ & Methodology & MSE & SNR(dB) & PSNR (dB) \\
\hline \multirow[t]{3}{*}{100} & MLPT & 0.0012 & 31.24 & 29.20 \\
\hline & EMD & 0.0244 & 17.27 & 16.13 \\
\hline & HYBRID MLPT-EEMD & 0.0004 & 36.37 & 34.02 \\
\hline \multirow[t]{3}{*}{103} & MLPT & 0.0132 & 22.47 & 18.82 \\
\hline & EMD & 0.0874 & 13.1 & 10.63 \\
\hline & HYBRID & 0.0044 & 27.84 & 23.88 \\
\hline \multirow[t]{3}{*}{104} & MLPT & 0.0350 & 16.26 & 14.55 \\
\hline & EMD & 0.1297 & 9.250 & 8.887 \\
\hline & HYBRID MLPT-EEMD & 0.0140 & 22.84 & 20.42 \\
\hline \multirow[t]{3}{*}{105} & MLPT & 0.0475 & 14.28 & 13.22 \\
\hline & EMD & 0.0864 & 11.04 & 10.63 \\
\hline & HYBRID MLPT-EEMD & 0.0265 & 17.46 & 15.94 \\
\hline \multirow[t]{3}{*}{106} & MLPT & 0.0292 & 18.94 & 15.34 \\
\hline & EMD & 0.3441 & 5.382 & 4.632 \\
\hline & HYBRID MLPT-EEMD & 0.0042 & 28.26 & 23.72 \\
\hline \multirow[t]{3}{*}{115} & MLPT & 0.0128 & 22.62 & 18.92 \\
\hline & EMD & 0.3080 & 5.811 & 5.113 \\
\hline & HYBRID & 0.0077 & 25.80 & 21.12 \\
\hline \multirow[t]{3}{*}{215} & MLPT & 0.0082 & 21.84 & 20.81 \\
\hline & EMD & 0.0209 & 17.31 & 16.79 \\
\hline & HYBRID & 0.0066 & 22.93 & 21.79 \\
\hline
\end{tabular}

Table 2. Comparative analysis

\begin{tabular}{|c|c|c|c|c|}
\hline Authors & $\begin{array}{c}\text { White Gaussian noise } \\
(\mathbf{d B})\end{array}$ & Method & $\begin{array}{c}\text { SNR } \\
(\mathbf{d B})\end{array}$ & MSE \\
\hline Wang, J., [11] & 20 & $\begin{array}{c}\text { Convolutional And Modified Elman Neural } \\
\text { Network }\end{array}$ & - & 0.0169 \\
\hline $\begin{array}{c}\text { Dengyong } \\
\text { Zhang [13] }\end{array}$ & 20 & EMD & 5.43 & 0.0024 \\
\hline $\begin{array}{c}\text { Ankita Mishra } \\
{[14]}\end{array}$ & 10 & $\begin{array}{c}\text { S-Transform Based Time-Frequency Filtering } \\
\text { Approach }\end{array}$ & 13.82 & 0.0287 \\
\hline Proposed & 10 & Hybrid MLPT-EEMD & 31.06 & 0.0007 \\
\cline { 2 - 5 } & 20 & & 25.93 & 0.0012 \\
\hline
\end{tabular}

proved by adding white Gaussian noise to each data series. The results of the hybrid MLPT-EEMD technique give better SNR values when compared to the existing empirical mode decomposition technique. The proposed hybrid MLPT-EEMD obtained SNR values of $25.93 \mathrm{~dB}$ better compared to the existing empirical mode decomposition technique (EMD) of $5.43 \mathrm{~dB}$, EMD with adaptive switching mean filter obtained $9.135 \mathrm{~dB}$ and S-Transform based TimeFrequency filtering approach obtained $13.82 \mathrm{~dB}$. However, noise properties were continuous and required a model built from scratch. Building such a model creates room for readjustment and fine-tuning. Thus, in the future, a model should be built to overcome the computation space and time issues.

\section{Conflicts of interest}

The authors declare no conflict of interest.

\section{Author contributions}

The paper background work, conceptualization, methodology, dataset collection, implementation, result analysis and comparison, preparing and editing draft, visualization have been done by first author. The supervision, review of work and project administration, has been done by second author.

\section{References}

[1] S. Saxena, R. Jais, and M. K. Hota, "Removal of Powerline Interference from ECG Signal using FIR, IIR, DWT and NLMS Adaptive Filter", In: Proc. of International Conference on Communication and Signal Processing (ICCSP), Chennai, India, pp. 0012-0016, 2019.

[2] M. Rakshit, and S. Das, "An efficient ECG denoising methodology using empirical mode 
decomposition and adaptive switching mean filter", Biomedical Signal Processing and Control, Vol. 40, pp. 140-148, 2018.

[3] G. Singh, G. Kaur, and V. Kumar, "ECG denoising using adaptive selection of IMFs through EMD and EEMD", In: Proc. of IEEE 2014 International Conference on Data Science \& Engineering (ICDSE), Kochi, India, pp. 228231, 2014.

[4] P. Singh, S. Shahnawazuddin, and G. Pradhan, "An Efficient ECG Denoising Technique Based on Non-local Means Estimation and Modified Empirical Mode Decomposition", Circuits, Systems, and Signal Processing, Vol. 37, No. 10, pp. 4527-4547, 2018.

[5] Y. Xu, M. Luo, T. Li, and G. Song, "ECG Signal De-noising and Baseline Wander Correction Based on CEEMDAN and Wavelet Threshold", Sensors, Vol. 17, p. 2754, 2017. doi:10.3390/s17122754

[6] X. Wan, H. Wu, F. Qiao, F. Li, Y. Li, Y. Yan, and J. Wei1, "Electrocardiogram Baseline Wander Suppression Based on the Combination of Morphological and Wavelet Transformation Based Filtering", Computational and Mathematical Methods in Medicine, Vol. 2019, No. 7, p. 7196156, 2019. https://doi.org/10.1155/2019/7196156

[7] Y. Luo, R. H. Hargraves, A. Belle, O. Bai, X. Qi, K. R. Ward, M. P. Pfaffenberger, and K. Najarian, "A Hierarchical Method for Removal of Baseline Drift from Biomedical Signals: Application in ECG Analysis", The World Scientific Journal, Vol. 2013, p. 896056, 2013. http://dx.doi.org/10.1155/2013/896056.

[8] R. Verma, R. Mehrotra, and V. Bhateja, "A New Morphological Filtering Algorithm for PreProcessing of Electrocardiographic Signals", In: Proc. of the Fourth International Conf. on Signal and Image Processing (ICSIP), Coimbatore, India, pp. 193-201, 2012.

[9] L. Yao, and Z. Pan, "A new method based CEEMDAN for removal of baseline wander and powerline interference in ECG signals", Optik, Vol. 223, p. 165566, 2020.

[10] A. Safari, H. D. Hesar, M. Mohebbi, and F. Faradji, "A Novel Method for R-peak Detection in Noisy ECG Signals Using EEMD and ICA", In: Proc. of $23^{\text {rd }}$ Iranian Conf. on Biomedical Engineering and $20161^{\text {st }}$ International Iranian Conf. on Biomedical Engineering (ICBME), Tehran, Iran, pp. 155-158, 2016.

[11] J. Wang, "A deep learning approach for atrial fibrillation signals classification based on convolutional and modified Elman neural network", Future Generation Computer Systems, Vol. 102, pp. 670-679, 2020.

[12] M. Murugappan, L. Murugesan, S. Jerritta, and H. Adeli, "Sudden Cardiac Arrest (SCA) Prediction Using ECG Morphological Features", Arabian Journal for Science and Engineering, pp. 1-15, 2020.

[13] D. Zhang, S. Wang, F. Li, S. Tian, J. Wang, X. Ding, and R. Gong, "An Efficient ECG Denoising Method Based on Empirical Mode Decomposition, Sample Entropy, and Improved Threshold Function", Wireless Communications and Mobile Computing, Vol. 2020, p. 8811962, 2020. https://doi.org/10.1155/2020/8811962

[14] A. Mishra, S. S. Sahu, R. Sharma, and S. K. Mishra, "Denoising of Electrocardiogram Signal Using S-Transform Based Time-Frequency Filtering Approach", Arabian Journal for Science and Engineering, Vol. 46, pp. 95159525, 2021.

[15] G. Moody, "A new method for detecting atrial fibrillation using R-R intervals", Computers in Cardiology, Vol. 10, pp. 227-230, 1983. 